OPEN ACCESS

Edited by:

Mark A. Elgar

The University of Melbourne, Australia

Reviewed by:

Michael Griesser,

University of Zurich, Switzerland

Dustin J. Penn,

University of Veterinary Medicine

Vienna, Austria

*Correspondence:

Michael J. Sheehan

msheehan@cornell.edu

Specialty section: This article was submitted to

Social Evolution,

a section of the journa

Frontiers in Ecology and Evolution

Received: 28 June 2019 Accepted: 19 December 2019

Published: 15 January 2020

Citation:

Tumulty JP and Sheehan MJ (2020)

What Drives Diversity in Social

Recognition Mechanisms?

Front. Ecol. Evol. 7:517.

doi: 10.3389/fevo.2019.00517

\section{What Drives Diversity in Social Recognition Mechanisms?}

\author{
James P. Tumulty and Michael J. Sheehan* \\ Department of Neurobiology and Behavior, Cornell University, Ithaca, NY, United States
}

Recognition allows animals to categorize social partners and differentiate among them in adaptive ways. Recognition systems are a fundamental component of social interactions, and a major goal for behavioral and evolutionary ecology is to understand the factors that influence the diversity of traits involved in social recognition across species and contexts. Here we argue that recognition is best understood as the interaction between a population of diverse senders and receivers with different perspectives and experiences. Receivers vary in the extent to which they agree on the category membership of senders and this variation is a key parameter that may explain the diverse evolutionary pressures shaping recognition systems. High receiver agreement (e.g., sex recognition) should favor uniformity in signals and innate recognition templates in receivers, while low receiver agreement (e.g., neighbor recognition) should tend to favor diversity in signals and flexible learning in receivers. Further, variation in how specifically receivers categorize senders may constrain the evolution of signals that need to function for multiple audiences. It remains an open question how receivers integrate multiple signals of different types of social categories. By framing recognition systems in a population context we hope this perspective will help spur new efforts to model and empirically investigate the mechanisms underlying the diversity of recognition systems across animals.

Keywords: individual recognition, class-level recognition, template, signal evolution, communication, social cognition, phenotypic diversity

\section{INTRODUCTION}

Animals need to categorize individuals they encounter to navigate their social environments. They do so using signals or cues produced by those individuals, and a social category consists of all of the individuals that are grouped together by a receiver. Examples of social categories include "my offspring" or "my territory neighbor." While receivers of a given species often have shared social categories of interest, they may disagree on who belongs in those categories (e.g., different territory holders will have different neighbors). They may also differ in which social categories they attend to at any given time. Our argument in this paper is that variation in receiver agreement about sender categorization has the potential to explain a great deal of the diversity in mechanisms of social recognition, in terms of variation in signals, and the mechanisms by which receivers classify signal variation.

A recognition system involves an interaction between a sender and a receiver of a communication signal or cue (Box 1; Sherman et al., 1997; Mateo, 2004). Recognition occurs when a receiver's perception of a signal or cue matches an internal representation ("template") of that signal or cue in the receiver's nervous system (Stoddard, 1996; Ryan and Rand, 2001; Bee, 2006). The receiver responds based on how it has categorized the signal, and the nature of the receiver's 
BOX 1 | Components of recognition systems.

1) Production: senders produce a signal or cue

2) Perception: receivers perceive a signal or cue and compare it to a template of that signal or cue in their nervous system.

a) Template acquisition: templates of signal or cue properties are either innate or learned

b) Categorization: the cognitive association of templates with relevant social categories

3) Action: receivers respond appropriately based on whether perception of a signal or cue matches their template.

response enacts fitness consequences for both the sender and the receiver (Reeve, 1989; Sherman et al., 1997; Liebert and Starks, 2004). Diverse mechanisms enable recognition across animal species, reflecting a variety of evolutionary trajectories of recognition system evolution. For example, signals vary from relatively uniform to highly variable among individuals (e.g., Buckley and Buckley, 1970; Gerhardt, 1991; Tibbetts, 2004). Further, receivers vary in the extent to which they rely on learned vs. innate templates in recognizing and responding to signals, and they vary in the specificity and complexity of their cognitive representations of social partners (Tibbetts and Dale, 2007; Miller and Bee, 2012; Wiley, 2013; Yorzinski, 2017). A remaining challenge for research on social recognition is to identify sources of selection that shape the evolution of traits that compose recognition systems.

Historically, research on social recognition has focused on the function and contexts of recognition, as well as the cognitive abilities of receivers in recognizing social partners (Box 2). Theoretical treatments of the evolution of recognition signals often consider average receivers in specific social contexts (e.g., Crozier, 1986; Beecher, 1989; Johnstone, 1997; Dale et al., 2001; Sheehan et al., 2017), but social recognition occurs among populations of diverse senders, and receivers with different experiences and motivations. This population perspective is important because receivers often vary in their responses based on, for example, morphology (Gill et al., 2013) or experience (Tanner et al., 2019), and this variation can impact signal evolution. Here we take a recognition systems approach (Boxes 1,2) to explore how variation in receiver agreement about how to categorize senders provides an important and overarching framework for explaining the diversity of recognition traits in both senders and receiver across a range of social and sexual contexts. Specifically, the extent to which receivers agree on how to categorize sender phenotypes is a key variable that may explain similarities and differences among diverse recognition systems.

\section{RECEIVER AGREEMENT FRAMEWORK}

We consider two axes of variation in agreement of sender categories for populations of receivers. First, receivers vary in the extent to which they agree on the category membership of
BOX 2 | Frameworks for classifying diversity in social recognition.

\section{Context approaches}

Recognition is commonly classified based on context or function. There are many examples of terms used to classify recognition based on context, including recognition of neighbors, nest-mates, group members, parentoffspring, mates, kin, castes, etc. Researchers using this terminology are often interested the adaptive value and social context of recognition. Kin recognition, in particular, has its own rich history of research and terminology for different mechanisms of recognition (reviewed in Penn and Frommen, 2010).

\section{Cognition-based approaches}

These approaches are primarily motivated by an interest in understanding receiver cognitive abilities and social intelligence. A point of considerable discussion and disagreement in this literature is the criteria needed to demonstrate individual recognition as opposed to a more generalized classlevel recognition (e.g., Halpin, 1986; Gheusi et al., 1994; Tibbetts and Dale, 2007; Johnston, 2008; Steiger and Müller, 2008). The distinction hinges on the specificity and complexity of an animals' cognitive representations of social partners and often seems aimed at finding examples that most closely approach our own capacities for individual recognition as humans. Class-level recognition is generally defined as occurring when a receiver recognizes a sender as belonging to a particular "class" or social category (e.g., neighbor vs. stranger, offspring vs. un-related young), but does not discriminate between individuals within a class (e.g., if a parent cares for all of its offspring equally). Individual recognition is commonly defined as when receivers can discriminate between individuals, even within a class, however varying definitions of individual recognition persist.

\section{Recognition systems approaches}

Recognition involves communication between a sender and a receiver of a communication signal or cue (see Box 1). Classifying the diversity in traits that underlie recognition can allow for the identification of recognition mechanisms that are shared across contexts and taxa as well as the evolutionary forces that shape these traits (Sherman et al., 1997; Tibbetts and Dale, 2007).

senders. In other words, do all receivers in a population agree that senders expressing trait $X$ belong to category $Y$ ? At one extreme, all receivers will agree on the category membership of senders. For example, adults of sexually dimorphic species express traits that are indicative of male or female. Red cardinals can be unambiguously classified as males by conspecifics. Though male and female cardinals may differ in how they respond to the category of "male," and females could be further interested in assessing fine variation in red coloration, the category of "male" is agreed upon. Situations in which receivers have high agreement on membership in social categories would include recognition of species, sex, age-class, and caste (Figure 1). These are objective social categories in that membership in such categories is intrinsic to senders and not dependent on receiver experience.

At the other extreme, receivers have low to no agreement on the category membership of senders. Consider a neighborhood where many individuals are defending territories across a landscape. Territory holders often recognize neighbors and only respond aggressively to strangers, producing a phenomenon called the "dear enemy effect" (Fisher, 1954; Wilson, 1975; Tumulty, 2018). Even if every territory holder recognizes neighbors, they won't agree on which individuals belong to this social category. In other words, one receiver's neighbor is 


\section{Population of signaling animals Receiver \#1's perspective Receiver \#2's perspective}

A Low receiver agreement: neighbor recognition in territorial frogs

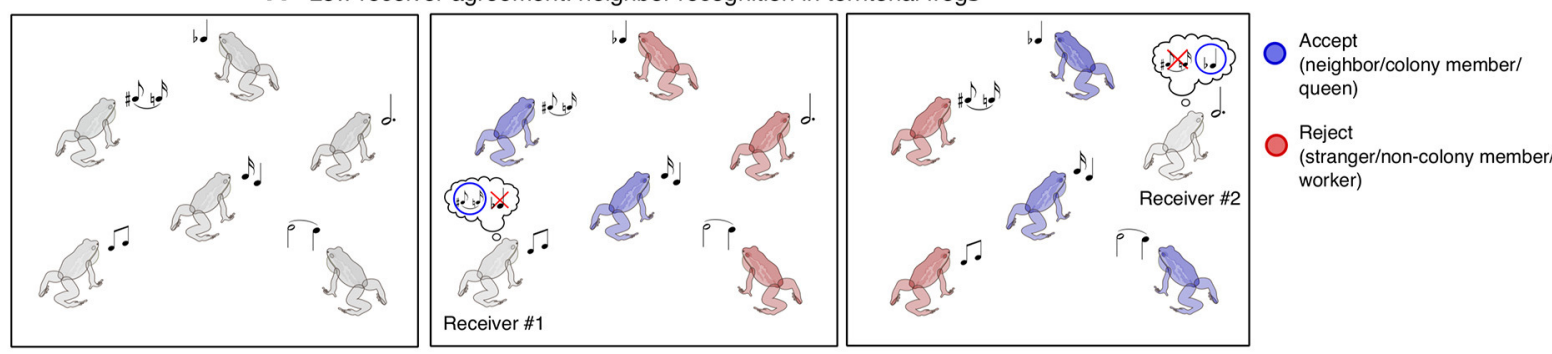

B Moderate receiver agreement: colony recognition in ants

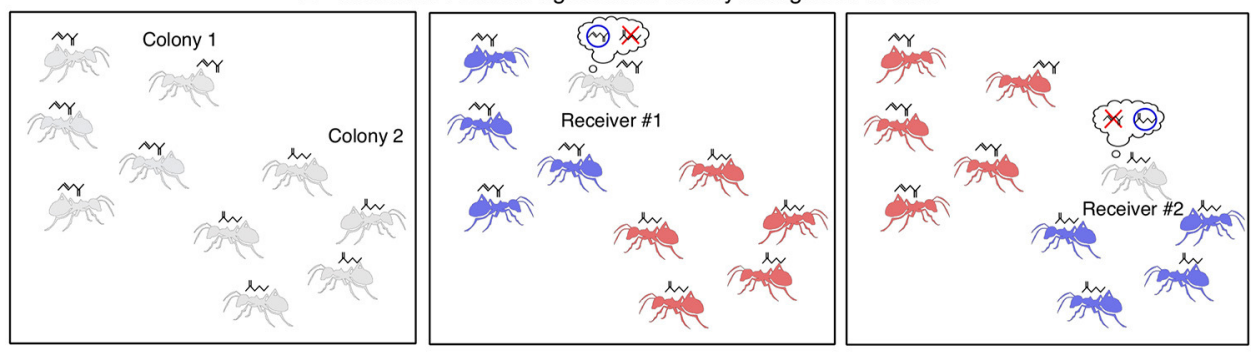

C High receiver agreement: queen recognition in ants
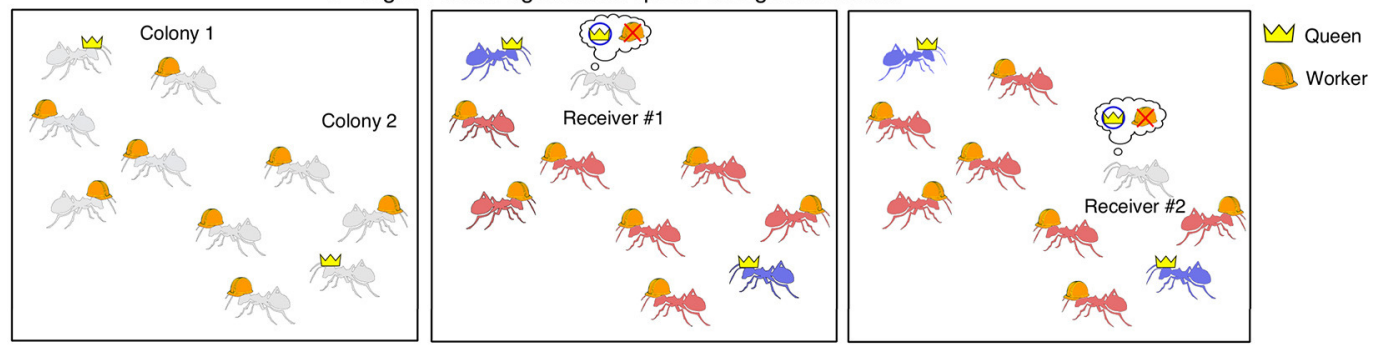

FIGURE 1 | Among recognition systems, receivers vary in the extent to which they agree on the category membership of senders. (A) In a population of territorial frogs, individuals are categorized as either neighbors, or strangers depending on their spatial proximity to a particular receiver. Receivers thus have low agreement on category membership and individuals must have individually distinctive traits (e.g., calls represented by musical notes in this example) to be recognized as members of such egocentric categories. Note: for simplicity, only two calls are shown in the receiver thought bubbles. (B) All members of a colony of ants share the same cuticular hydrocarbon profile, which differs between colonies. The decision of whether to accept an individual as a colony member or reject it as a non-colony member depends on the colony to which a receiver belongs. At the level of the population, there are moderate levels of receiver agreement because all members of the same colony agree on who belongs in which categories, but members of different colonies disagree. (C) Whether an individual is a queen (represented by a crown) or a worker (hard hat) is an objective social category that does not depend on the perspective of a receiver, resulting in high agreement about category membership among a population of receivers.

another receiver's stranger. Therefore, a population of receivers will have low agreement on which members of the population belong in the neighbor vs. stranger categories. As such, it is not possible for a single phenotype to be associated with "neighbor" or "stranger" (Figure 1). Situations with low receiver agreement include recognition of mates, parents, offspring, and neighbors. These are egocentric social categories because assignment to a particular category depends on the experience of a particular receiver and not on intrinsic features of the sender.

The second axis of variation we explore is the specificity of categories that receivers attend to. The specificity of social categories can be considered hierarchically based on the number of individuals that belong to categories (Wiley, 2013). The most general category includes all individuals of a given species, while the most specific is a single individual. Intermediate levels of specificity range from "my offspring" which may include a handful of individuals, to "colony member" which may include hundreds or thousands of individuals in the case of social insects. Different receivers in a population may be interested in different levels of this continuum simultaneously. For example, a parent may need to categorize its offspring as individuals, or perhaps more generally as "my offspring" vs. "not my offspring." While this need for categorization may be true of all parents in a population, non-parent receivers would not need to know whose offspring are whose, but they may still care to know that a sender is immature. In this example, a given juvenile may be categorized as "my offspring" by its parent, "not my offspring" by an unrelated parent, or more generically as "juvenile" depending on the receiver in question. Senders may produce signals or 
cues that that allow for recognition and categorization across multiple hierarchical layers due to the variation in specificity of recognition among receivers within a population.

\section{IMPLICATIONS TO THE EVOLUTION OF RECOGNITION MECHANISMS}

Understanding the extent to which receivers agree about the categorization of senders provides a novel framework for thinking about both the mechanisms and evolution of recognition systems. Here, we focus on situations in which senders and receivers have shared evolutionary interests, but we also briefly highlight how conflicting interests between senders and receivers may shape recognition systems.

\section{Receivers Vary in the Extent to Which They Agree on Category Membership Consequences for Senders}

When receivers agree on categorization, similarity among senders should be favored as uniformity within a category will facilitate recognition across receivers (Figure 2). For signals with a genetic basis, such uniformity can be the result of stabilizing selection within categories and divergence between categories. There are countless examples of signals that have likely been shaped by recognition in this way, including calls used for species recognition in gray treefrogs (Hyla versicolor, Gerhardt, 1991), throat color signals of mating strategy in side-blotched lizards (Uta stansburiana, Sinervo and Lively, 1996), visual signals of sexual receptivity in female sticklebacks (Gasterosteus aculeatus, Rowland et al., 1991), pheromones used for sex recognition in crayfish (Procambarus clarkii, Ameyaw-Akumfi and Hazlett, 1975; Hazlett, 1985), and cuticular hydrocarbons used for caste recognition in ants (Aphaenogaster senilis, Ruel et al., 2013). For signals that are more plastic, uniformity can be achieved through homogenization among category members. Colony-specific odors used for nest-mate recognition in social insects develop in just this way (Gamboa et al., 1986; Breed et al., 1988; Breed and Stiller, 1992). Both of these processes would result in senders within a category having similar traits (i.e., homogenous subgroups; Barrows et al., 1975). While there may be many inadvertent cues of category membership that are produced because of a shared genetic or developmental basis of particular categories (e.g., males and females often differ in body size for reasons unrelated to sex recognition), if senders benefit from being recognized, selection should favor the elaboration of signals that facilitate recognition.

If receivers do not agree on which individuals belong to categories, they must recognize social partners using individually distinctive phenotypic traits and associate these traits with social categories (Figure 2). For example, as discussed above, there cannot be inherent phenotypic traits associated with categories such as "neighbor" or "stranger," because such categories are egocentric and dependent on receiver experience. Very few territory owners will share the same neighbors, so if territory owners are to recognize neighbors, they must do so using individually distinctive phenotypic traits of neighbors (Figure 1). Individuals may often vary due to genetic and developmental differences unrelated to recognition, but provided being recognized is beneficial, selection should favor greater phenotypic diversity in senders when receivers do not agree on category membership. Such diversity can result from negative frequency dependent selection in which individuals with rare, recognizable phenotypes have higher fitness than individuals with common phenotypes (Sheehan and Tibbetts, 2009; Tibbetts et al., 2017). Signals in paper wasps (Polistes fuscatus; Sheehan and Tibbetts, 2010), house mice (Mus musculus; Sheehan et al., 2016), and humans (Homo sapiens; Sheehan and Nachman, 2014) show patterns of selection for identity signaling, as do signals across species of swallows (Medvin et al., 1993), bats (Wilkinson, 2003), marmots (Pollard and Blumstein, 2011), and penguins (Aubin and Jouventin, 2002).

\section{Consequences for Receivers}

Similarity among senders means that receivers can have shared templates that are used to recognize members of relevant categories (Figure 2). Where template similarity is favored, innate templates are possible. This is because the meaning of a signal of category membership is stable across generations. In such situations, learning is not required each generation and coevolution between senders and receivers can select for signals that are reliably associated with category membership and templates that enable receivers to respond appropriately to such signals. Innate templates of agreed-upon categories are likely extremely common in nature. Some examples include recognition of cues of sexual receptivity in rats (Rattus norvegicus, Landauer et al., 1977) and mammary pheromones in newborn rabbits (Oryctolagus cuniculus, Schneider et al., 2016).

Recognition templates must be learned when receivers disagree on category membership of senders. This is because such categories are egocentric with respect to a particular receiver (Figure 2). Templates could be acquired through imprinting if senders are encountered early in life in a predictable context and their membership in that category does not change within a lifetime (e.g., parent imprinting in ducks and geese, reviewed in Shettleworth, 2009). However, if category membership changes throughout a lifetime, templates must continue to be acquired, or updated. For example, the identity of a neighbor is seldom fixed during a lifetime and is determined based on where an animal establishes a territory and who settles nearby. Further, territory occupancy may change over time, and residents often have multiple neighbors (Stoddard, 1996; Wiley, 2013). In these situations, animals must be able to form multiple recognition templates over the course of their lives, and potentially update and modify these templates. For example, territorial white-crowned sparrows (Brooks and Falls, 1975; Zonotrichia albicollis, Baker et al., 1981), and bullfrogs (Rana catesbeiana, Bee and Gerhardt, 2002) likely learn to recognize neighbors by habituating to their neighbors' vocalizations and territory locations, allowing them to discriminate between multiple neighbors and create new neighbor templates if new neighbors arrive. 
A High receiver agreement

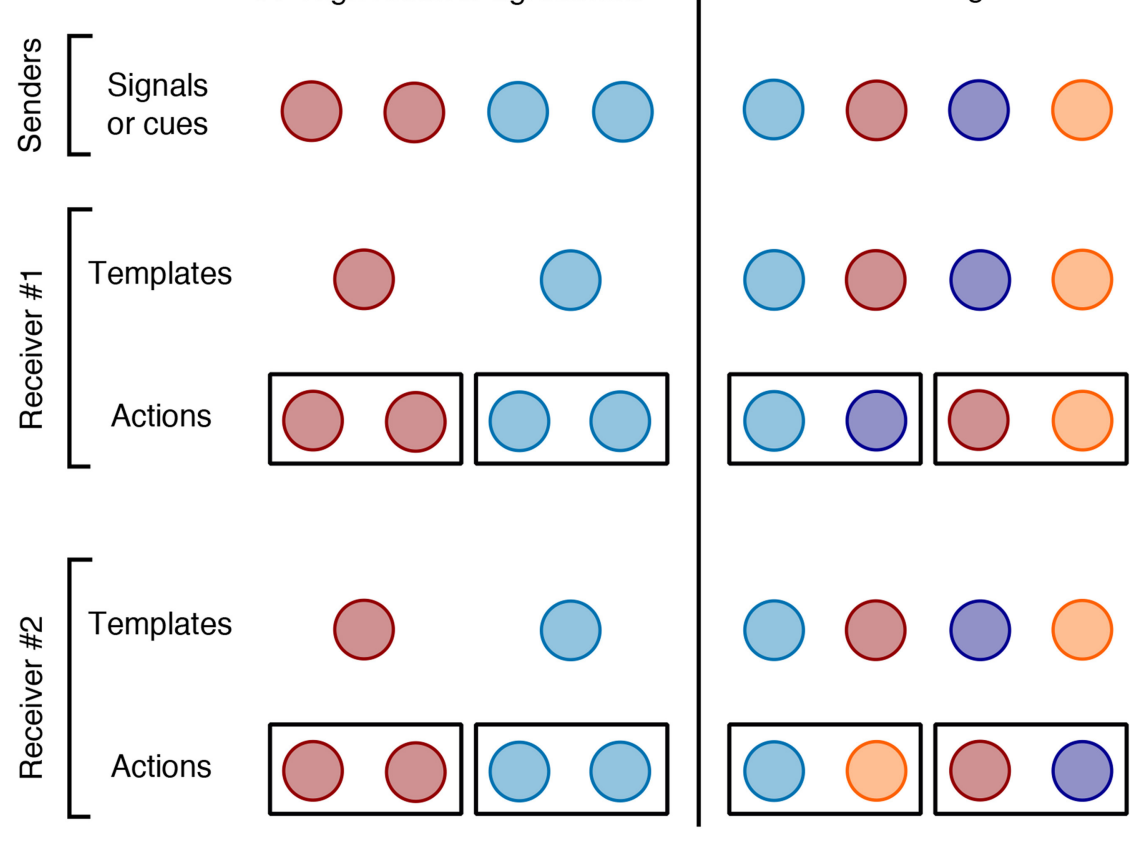

FIGURE 2 | The representation and grouping of sender phenotypes (colors) as (1) signals or cues produced by senders, (2) templates in a receiver's nervous system, and (3) receiver actions (e.g., accept or reject). Each dot represents a hypothetical individual, different colors represent different phenotypic signals or cues used for recognition, and boxes surround individuals who are treated as belonging to the same social category by a receiver's actions. (A) when receivers agree on category membership (e.g., sex recognition), senders share signals or cues associated with a certain category, and receivers possess one template per category that can be used to recognize any individual that they encounter who is expressing that signal or cue. Recognition is not dependent on receiver experience so all receivers should associate the same phenotypes with the same categories. (B) When receivers disagree on category membership (e.g., neighbor recognition), signals or cues and templates are individual-specific but actions need not be. Receivers in population can share the same categories but they disagree on who belongs in those categories so they must use individually-distinctive traits to assign senders to categories.

\section{When Senders and Receivers Have Conflicting Interests}

The predictions outlined above are for situations in which senders and receivers both benefit from recognition. However, when senders do not benefit by being recognized, we would generally expect the opposite patterns to emerge. When receivers agree, selection may favor diversity in senders to inhibit recognition and favor learning in receivers. This process seems to underlie apostatic selection as prey evolve polymorphisms to avoid detection by predator search images (Bond and Kamil, 1998). Conversely, when receivers disagree about category membership, senders could cause confusion by evolving uniformity. This may be important in instances of paternity confusion. Fathers are interested in differentiating their own offspring from other offspring, but detection would be costly to extra-pair offspring, selecting against identity signaling by chicks (Kempenaers and Sheldon, 1996).

\section{Receivers Vary in the Specificity of Categorization \\ Consequences for Senders}

A population of receivers that are interested in different levels of social categories may constrain the evolution of signals. Many receivers attending to and responding to signals or cues associated with social categories impose selection on senders, and the benefit of communicating information that all receivers could agree upon at one level (e.g., "male of my species") could favor uniformity that may then constrain the diversity of traits at another level of categorization (e.g., "individual" or "neighbor"). Senders can potentially solve this problem in two ways. First, senders can adjust the types of signals they provide in different contexts or life stages if the relative importance of different groups of receivers and the costs and benefits of recognition also vary across life stages and contexts. The plumage patterns of royal terns (Thalasseus maxima) provides an interesting example. Mobile young are reared in large colonies making it potentially challenging for parents to locate their offspring. Chicks have highly variable plumage coloration and patterning (Buckley and Buckley, 1970) that facilitates offspring recognition by parents (Buckley and Buckley, 1972). However, at later life stages, juveniles, non-breeding adults, and breeding adults all have distinct plumage patterns that are associated with their age and breeding status (Buckley and Buckley, 2002). Whereas, receiver disagreement appears to be an important force shaping plumage identity signals in young terns, adult breeding plumage is relatively uniform among senders within a given social category, suggesting that the receiver agreement in categorization is high at later life stages. The second potential solution is to use multiple signals components or modalities to convey information. We would expect this solution to arise in situations 
where different receivers are interested in different levels of categorization simultaneously. This solution is analogous to the "multiple messages" hypothesis in animal communication (Gerhardt, 1992; Johnstone, 1996), which posits that different signal components convey different types of information about the sender. For example, many vocalizations encode information about identity, age, and sex, such as alarm calls of yellow-bellied marmots (Marmota flaviventri, Blumstein and Munos, 2005) or bleats of giant pandas (Ailuropoda melanoleuca, Charlton et al., 2009). Scent marks also frequently contain multiple tiers of social information, as has been shown for black rhinoceros (Diceros bicornis, Linklater et al., 2013) and house mice (Mus musculus, Hurst and Beynon, 2004; Sheehan et al., 2019). The role that populations of receivers play in shaping these signals by responding to different levels of social information in these signals simultaneously is very much an open question.

\section{Consequences for Receivers}

Because senders are evolving to provide information to a population of receivers that will vary in extent of interest and agreement on categorization, receivers should be equipped to assess a wide range of sender phenotypes that will correspond to both innate and learned templates. This raises the question of how receivers integrate information that corresponds to multiple templates and categories when assessing a sender. An individual's identity is made up of many different categories that vary in specificity, including species, sex, and age. As reviewed above, these features can often be encoded within a single complex trait such as a vocalization, scent, or color pattern. It is clear that receivers can extract information at different levels of specificity from the same signals. For example, field sparrows use variation in song frequency to recognize both species and individuals (Spizella pusilla, Nelson, 1989). Further, experiments in domestic horses (Equus caballus, Proops et al., 2009) and rhesus macaques (Macaca mulatta, Sliwa et al., 2011) demonstrate that receivers sometimes integrate signals from different modalities (visual and auditory) to recognize individuals. But how do receivers integrate information about different levels of specificity in recognition? For example, when a receiver recognizes a sender as an individual, do the features that make the sender recognizable as part of broader, agreed-upon categories like species and sex form part of the template for that individual? Or are templates for different levels of specificity distinct, with receivers using simpler decision rules for discriminating broader categories and attending to more specific levels of categorization only when it is beneficial to do so? Understanding the hierarchy of receiver categorization is important to uncovering the mechanisms by which recognition systems work in the complex social settings found in real animal populations. This is currently an unresolved question, but its answer will reveal how receivers integrate information across levels of social categories.

\section{IMPLICATIONS FOR OTHER CONTEXTS}

Recognition is a fundamental component of animal lives beyond their social interactions and variation in receiver agreement may have implications to other contexts as well. Here we briefly discuss some of these potential implications. First, foraging is a context that is often mediated by communication (Bradbury and Vehrencamp, 2011). For example, aposematic animals benefit by advertising their toxicity to would-be predators and predators benefit by recognizing aposematic signals as indicators of toxicity (Rojas et al., 2015). Müllerian mimicry, whereby two different toxic species converge on the same aposematic phenotype (e.g., Kapan, 2001; Symula et al., 2001), may represent an example of selection for uniformity driven by high receiver agreement since receivers (predators) possess or develop one template for the objective category "toxic prey" and thereby impose selection on senders that are members of this category to label themselves as such with shared signals. Pollination syndromes-similar suites of shared floral traits among different species that attract the same pollinators (Fenster et al., 2004)—represent a similar example of convergent evolution of signals in response to shared receiver templates. Examples of receiver disagreement in foraging contexts are perhaps less common because there are fewer opportunities for repeated interactions between the same individuals. But, by generalizing our framework to repeated interactions that an individual has with members of a given species that it forages on, floral constancy may represent an example of low receiver agreement. Floral constancy occurs when pollinators demonstrate short-term learned preferences for certain flowers as a result of associating a reward (nectar) with signals from that particular flower (e.g., color, odor) (Schiestl and Johnson, 2013). This preference is egocentric since not all foragers will express the same preference (Heinrich, 1976), and it is thought to be a source of selection for floral distinctiveness (Schiestl and Johnson, 2013). A second context in which this receiver agreement framework seems relevant is in the importance of learning mate choice preferences. When preferences are genetically determined, a population of receivers agree on the traits that indicate high quality mates, and thus impose stabilizing or directional selection on those traits. However, in some cases, receivers can have low preference agreement if preferences for traits that are experienced early in life are learned, a phenomenon termed "sexual imprinting" (Verzijden et al., 2012). This phenomenon can be a source of selection for signal diversity, for example, by helping to maintain reproductive isolation between closely related sympatric species (Verzijden and Ten Cate, 2007) or leading to the stable coexistence of polymorphisms under some conditions (Yang et al., 2019).

\section{CONCLUSIONS AND FUTURE DIRECTIONS}

Here, we have argued that recognition is a multiparty process in which senders and receivers communicate with each other in a population. We argue for taking into account the diversity of receiver perspectives, as doing so can provide new insights into the evolution of traits mediating recognition. Notably, this perspective applies to a range of social categorization 
and recognition contexts, providing an overarching framework to consider how receiver behavior and sender phenotypes interact and coevolve. Whether or not receivers agree on how to categorize a sender should determine the selection pressures shaping patterns of diversity in signals. At the same time, agreement among receivers opens of the possibility of innate templates, whereas disagreement requires learned templates. Not all receivers will be concerned with identifying senders based on the same sorts of social categories, which may constrain the evolution of sender traits. This framework highlights the need for future models of recognition systems to consider the diversity of receiver perspectives and for empirical studies to probe the constraints on sender traits and receiver integration of innate and learned templates when assessing senders.

\section{REFERENCES}

Ameyaw-Akumfi, C., and Hazlett, B. A. (1975). Sex recognition in crayfish Procambarus clarkii. Science 190, 1225-1226. doi: 10.1126/science. 1198111

Aubin, T., and Jouventin, P. (2002). How to vocally identify kin in a crowd: the penguin model. Adv. Study Behav. 31, 243-277. doi: 10.1016/S0065-3454(02)80010-9

Baker, M. C., Thompson, D. B., and Sherman, G. L. (1981). Neighbor/stranger song discrimination in white-crowned sparrows. Condor 83, 265-267. doi: $10.2307 / 1367322$

Barrows, E. M., Bell, W. J., and Michener, C. D. (1975). Individual odor differences and their social functions in insects. Proc. Natl. Acad. Sci. U.S.A. 72, 2824-2828. doi: 10.1073/pnas.72.7.2824

Bee, M. A. (2006). "Individual recognition in animal species", in The Encyclopedia of Language and Linguistics: Vol 2, ed N. Naguib (London: Elsevier Science), 617-626. doi: 10.1016/B0-08-044854-2/00817-8

Bee, M. A., and Gerhardt, H. C. (2002). Individual voice recognition in a territorial frog (Rana catesbeiana). Proc. R. Soc. London B Biol. Sci. 269, 1443-1448. doi: 10.1098/rspb.2002.2041

Beecher, M. D. (1989). Signalling systems for individual recognition: an information theory approach. Anim. Behav. 38, 248-261. doi: 10.1016/S0003-3472(89)80087-9

Blumstein, D. T., and Munos, O. (2005). Individual, age and sex-specific information is contained in yellow-bellied marmot alarm calls. Anim. Behav. 69, 353-361. doi: 10.1016/j.anbehav.2004.10.001

Bond, A. B., and Kamil, A. C. (1998). Apostatic selection by blue jays produces balanced polymorphism in virtual prey. Nature 395, 594-596. doi: $10.1038 / 26961$

Bradbury, J. W., and Vehrencamp, S. L. (2011). Principles of Animal Communication, 2nd Edn. Sunderland, MA: Sinauer Associates.

Breed, M. D., and Stiller, T. M. (1992). Honey bee, Apis mellifera, nestmate discrimination: hydrocarbon effects and the evolutionary implications of comb choice. Anim. Behav. 43, 875-883. doi: 10.1016/S0003-3472(06)8 0001-1

Breed, M. D., Williams, K. R., and Fewell, J. H. (1988). Comb wax mediates the acquisition of nest-mate recognition cues in honey bees. Proc. Natl. Acad. Sci. U.S.A. 85, 8766-8769. doi: 10.1073/pnas.85.22.8766

Brooks, R. J., and Falls, J. B. (1975). Individual recognition by song in whitethroated sparrows. II. Effects of location. Can. J. Zool. 53, 1412-1420. doi: $10.1139 / \mathrm{z} 75-170$

Buckley, P. A., and Buckley, F. G. (1970). Color variation in the soft parts and down of royal tern chicks. Auk 87, 1-13. doi: 10.2307/4083655

Buckley, P. A., and Buckley, F. G. (1972). Individual egg and chick recognition by adult royal terns (Sterna maxima maxima).

\section{AUTHOR CONTRIBUTIONS}

All authors contributed to the development of the ideas and writing and editing of the manuscript.

\section{FUNDING}

The authors were supported by the National Science Foundation (DEB 1750394) and the National Institutes of Health (1DP2GM128202-01).

\section{ACKNOWLEDGMENTS}

We thank members of the Sheehan lab and two reviewers for feedback on earlier versions of this manuscript.

Anim. Behav. 20, 457-462. doi: 10.1016/S0003-3472(72)8 0009-5

Buckley, P. A., and Buckley, F. G. (2002). "Royal Tern (Thalasseus maximus), version 2.0," in The Birds of North America, eds A. F. Poole and F. B. Gill (Ithaca, NY: Cornell Lab of Ornithology).

Charlton, B. D., Zhihe, Z., and Snyder, R. J. (2009). The information content of giant panda, Ailuropoda melanoleuca, bleats: acoustic cues to sex, age and size. Anim. Behav. 78, 893-898. doi: 10.1016/j.anbehav.2009. 06.029

Crozier, R. (1986). Genetic clonal recognition abilities in marine invertebrates must be maintained by selection for something else. Evolution 40, 1100-1101. doi: 10.1111/j.1558-5646.1986.tb00578.x

Dale, J., Lank, D. B., and Reeve, H. K. (2001). Signaling individual identity versus quality: a model and case studies with ruffs, queleas, and house finches. Am. Nat. 158, 75-86. doi: 10.1086/3 20861

Fenster, C. B., Armbruster, W. S., Wilson, P., Dudash, M. R., and Thomson, J. D. (2004). Pollination syndromes and floral specialization. Аnnu. Rev. Ecol. Evol. Syst. 35, 375-403. doi: 10.1146/annurev.ecolsys.34.011802. 132347

Fisher, J. (1954). "Evolution and bird sociality," in Evolution as a Process, eds J. Huxley, A. C. Hardy, and E. B. Ford (London: Allen \& Unwin), 71-83.

Gamboa, G. J., Reeve, H. K., Ferguson, I. D., and Wacker, T. L. (1986). Nestmate recognition in social wasps: the origin and acquisition of recognition odours. Anim. Behav. 34, 685-695. doi: 10.1016/S0003-3472(86)80053-7

Gerhardt, H. C. (1991). Female mate choice in treefrogs: static and dynamic acoustic criteria. Anim. Behav. 42, 615-635. doi: 10.1016/S0003-3472(05)80245-3

Gerhardt, H. C. (1992). Multiple messages in acoustic signals. Semin. Neurosci. 4, 391-400. doi: 10.1016/1044-5765(92)90047-6

Gheusi, G., Bluthé, R. M., Goodall, G., and Dantzer, R. (1994). Social and individual recognition in rodents: methodological aspects and neurobiological bases. Behav. Processes 33, 59-87. doi: 10.1016/0376-6357(94)90060-4

Gill, K. P., van Wilgenburg, E., Macmillan, D. L., and Elgar, M. A. (2013). Density of antennal sensilla influences efficacy of communication in a social insect. Am. Nat. 182, 834-840. doi: $10.1086 / 673712$

Halpin, Z. T. (1986). Individual odors among mammals: origins and functions. Adv. Study Behav. 16, 39-70. doi: 10.1016/S0065-3454(08)60187-4

Hazlett, B. A. (1985). Chemical detection of sex and condition in the crayfish Orconectes virilis. J. Chem. Ecol. 11, 181-189. doi: 10.1007/BF00988201

Heinrich, B. (1976). The foraging specializations of individual bumblebees. Ecol. Monogr. 46, 105-128. doi: 10.2307/1942246

Hurst, J. L., and Beynon, R. J. (2004). Scent wars: the chemobiology of competitive signalling in mice. BioEssays 26, 1288-1298. doi: 10.1002/bies. 20147 
Johnston, R. E. (2008). Individual odors and social communication. Individual recognition, kin recognition, and scent over-marking. Adv. Study Behav. 38, 439-505. doi: 10.1016/S0065-3454(08)00 009-0

Johnstone, R. A. (1996). Multiple displays in animal communication: "backup signals" and "multiple messages." Philos. Trans. R. Soc. B Biol. Sci. 351, 329-338. doi: 10.1098/rstb.1996.0026

Johnstone, R. A. (1997). Recognition and the evolution of distinctive signatures: when does it pay to reveal identity? Proc. R. Soc. B Biol. Sci. 264, 1547-1553. doi: 10.1098/rspb.1997.0215

Kapan, D. D. (2001). Three-butterfly system provides a field test of müllerian mimicry. Nature 409, 18-20. doi: 10.1038/35053066

Kempenaers, B., and Sheldon, B. C. (1996). Why do male birds not discriminate between their own and extra-pair offspring? Anim. Behav. 51, 1165-1173. doi: 10.1006/anbe.1996.0118

Landauer, M. R., Wiese, R. E., and Carr, W. J. (1977). Responses of sexually experienced and naive male rats to cues from receptive vs. nonreceptive females. Anim. Learn. Behav. 5, 398-402. doi: 10.3758/BF032 09586

Liebert, A. E., and Starks, P. T. (2004). The action component of recognition systems: a focus on the response. Ann. Zool. Fennici 41, 747-746.

Linklater, W. L., Mayer, K., and Swaisgood, R. R. (2013). Chemical signals of age, sex and identity in black rhinoceros. Anim. Behav. 85, 671-677. doi: 10.1016/j.anbehav.2012.12.034

Mateo, J. M. (2004). Recognition systems and biological organization: the perception component of social recognition. Ann. Zool. Fennici $41,729-745$

Medvin, M. B., Stoddard, P. K., and Beecher, M. D. (1993). Signals for parent-offspring recognition: a comparative analysis of begging calls of cliff swallows and barn swallows. Anim. Behav. 45, 841-850. doi: 10.1006/anbe.199 3.1105

Miller, C. T., and Bee, M. A. (2012). Receiver psychology turns 20: is it time for a broader approach? Anim. Behav. 83, 331-343. doi: 10.1016/j.anbehav.2011.11.025

Nelson, D. A. (1989). Song frequency as a cue for recognition of species and individuals in the field sparrow (Spizella pusilla). J. Comp. Psychol. 103, 171-6. doi: 10.1037/0735-7036.103.2.171

Penn, D. J., and Frommen, J. G. (2010). "Kin recognition: an overview of conceptual issues, mechanisms and evolutionary theory," in Animal Behaviour: Evolution and Mechanisms (Berlin; Heidelberg: Springer), 55-85. doi: 10.1007/978-3-642-02624-9_3

Pollard, K. A., and Blumstein, D. T. (2011). Social group size predicts the evolution of individuality. Curr. Biol. 21, 413-417. doi: 10.1016/j.cub.2011. 01.051

Proops, L., Mccomb, K., and Reby, D. (2009). Cross-modal individual recognition in domestic horses (Equus caballus). Proc. Natl. Acad. Sci. U.S.A. 106, 947-951. doi: $10.1073 /$ pnas.0809127105

Reeve, H. K. (1989). The evolution of conspecific acceptance thresholds. Am. Nat. 133, 407-435. doi: 10.1086/284926

Rojas, B., Valkonen, J., and Nokelainen, O. (2015). Aposematism. Curr. Biol. 25, R350-R351. doi: 10.1016/j.cub.2015.02.015

Rowland, W. J., Baube, C. L., and Horan, T. T. (1991). Signalling of sexual receptivity by pigmentation pattern in female sticklebacks. Anim. Behav. 42, 243-249. doi: 10.1016/S0003-3472(05)80555-X

Ruel, C., Hefetz, A., Cerdá, X., and Boulay, R. (2013). Recognition of caste and mating status maintains monogyny in the ant Aphaenogaster senilis. Behav. Ecol. Sociobiol. 67, 1295-1305. doi: 10.1007/s00265-013-1558-x

Ryan, M. J., and Rand, A. S. (2001). "Feature weighting in signal recognition and discrimination by túngara frogs," in Anuran Communication, ed M. J. Ryan (Washington, DC: Smithsonian Institution Press), 86-101.

Schiestl, F. P., and Johnson, S. D. (2013). Pollinator-mediated evolution of floral signals. Trends Ecol. Evol. 28, 307-315. doi: 10.1016/j.tree.2013.01.019

Schneider, N. Y., Piccin, C., Datiche, F., and Coureaud, G. (2016). Spontaneous brain processing of the mammary pheromone in rabbit neonates prior to milk intake. Behav. Brain Res. 313, 191-200. doi: 10.1016/j.bbr.2016. 07.014
Sheehan, M. J., Campbell, P., and Miller, C. H. (2019). Evolutionary patterns of major urinary proteins scent signals in house mice and relatives. Mol. Ecol. 28, 3587-3601. doi: 10.1111/mec.15155

Sheehan, M. J., Lee, V., Corbett-Detig, R., Bi, K., Beynon, R. J., Hurst, J. L., et al. (2016). Selection on coding and regulatory variation maintains individuality in major urinary protein scent marks in wild mice. PLoS Genet. 12: e1005891. doi: 10.1371/journal.pgen.10 05891

Sheehan, M. J., Miller, C., and Reeve, H. K. (2017). Identity signaling and patterns of cooperative behavior. Integr. Comp. Biol. 57, 580-588. doi: $10.1093 /$ icb/icx054

Sheehan, M. J., and Nachman, M. W. (2014). Morphological and population genomic evidence that human faces have evolved to signal individual identity. Nat. Commun. 5:4800. doi: 10.1038/ncomms5800

Sheehan, M. J., and Tibbetts, E. A. (2009). Evolution of identity signals: frequencydependent benefits of distinctive phenotypes used for individual recognition. Evolution 63, 3106-3113. doi: 10.1111/j.1558-5646.2009.00833.x

Sheehan, M. J., and Tibbetts, E. A. (2010). Selection for individual recognition and the evolution of polymorphic identity signals in Polistes paper wasps. J. Evol. Biol. 23, 570-577. doi: 10.1111/j.1420-9101.2009.01923.x

Sherman, P. W., Reeve, H. K., and Pfennig, D. W. (1997). "Recognition systems," in Behavioral Ecology: An Evolutionary Approach, eds J. R. Krebs and N. B. Davies (Oxford: Blackwell Science Ltd), 69-96.

Shettleworth, S. J. (2009). Cognition, Evolution, and Behavior. New York, NY: Oxford University Press.

Sinervo, B., and Lively, C. M. (1996). The rock-paper-scissors game and the evolution of alternative male strategies. Nature 380, 240-243. doi: $10.1038 / 380240 \mathrm{a} 0$

Sliwa, J., Duhamel, J.-R., Pascalis, O., and Wirth, S. (2011). Spontaneous voice-face identity matching by rhesus monkeys for familiar conspecifics and humans. Proc. Natl. Acad. Sci. U.S.A. 108, 1735-1740. doi: 10.1073/pnas.1008169108

Steiger, S., and Müller, J. K. (2008). "True" and "untrue" individual recognition: suggestion of a less restrictive definition. Trends Ecol. Evol. 23:355; author reply 356. doi: $10.1016 /$ j.tree.2008.01.014

Stoddard, P. K. (1996). "Vocal recognition of neighbors by territorial passerines," in Ecology and Evolution of Acoustic Communication in Birds, eds D. E. Kroodsma and E. H. Miller (Ithaca, NY: Cornell University Press), 356-34.

Symula, R., Schulte, R., and Summers, K. (2001). Molecular phylogenetic evidence for a mimetic radiation in Peruvian poison frogs supports a Müllerian mimicry hypothesis. Proc. Biol. Sci. 268, 2415-2421. doi: 10.1098/rspb. 2001.1812

Tanner, J. C., Garbe, L. M., and Zuk, M. (2019). When virginity matters: age and mating status affect female responsiveness in crickets. Anim. Behav. 147, 83-90. doi: 10.1016/j.anbehav.2018.11.006

Tibbetts, E. A. (2004). Complex social behaviour can select for variability in visual features: a case study in Polistes wasps. Proc. R. Soc. London B Biol. Sci. 271, 1955-1960. doi: 10.1098/rspb.2004.2784

Tibbetts, E. A., and Dale, J. (2007). Individual recognition: it is good to be different. Trends Ecol. Evol. 22, 529-537. doi: 10.1016/j.tree.2007.09.001

Tibbetts, E. A., Mullen, S. P., and Dale, J. (2017). Signal function drives phenotypic and genetic diversity: the effects of signalling individual identity, quality or behavioural strategy. Philos. Trans. R. Soc. B Biol. Sci. 372:20160347. doi: 10.1098/rstb.2016.0347

Tumulty, J. P. (2018). "Dear Enemy Effect," in Encyclopedia of Animal Cognition and Behavior, eds J. Vonk and T. Shackelford (Cham: Springer International Publishing), 1-4. doi: 10.1007/978-3-319-47829-6_693-1

Verzijden, M. N., and Ten Cate, C. (2007). Early learning influences species assortative mating preferences in Lake Victoria cichlid fish. Biol. Lett. 3, 134-136. doi: 10.1098/rsbl.2006.0601

Verzijden, M. N., Ten Cate, C., Servedio, M. R., Kozak, G. M., Boughman, J. W., and Svensson, E. I. (2012). The impact of learning on sexual selection and speciation. Trends Ecol. Evol. 27, 511-519. doi: 10.1016/j.tree.2012. 05.007

Wiley, R. H. (2013). Specificity and multiplicity in the recognition of individuals: implications for the evolution of social behaviour. Biol. Rev. 88, 179-195. doi: 10.1111/j.1469-185X.2012.00246.x 
Wilkinson, G. S. (2003). "Social and vocal complexity in bats", in Animal Social Complexity, eds F. B. de Waal and P. L. Tyack (Cambridge, MA: Harvard University Press), 322-341.

Wilson, E. O. (1975). Sociobiology: The New Synthesis. Cambridge, MA: Harvard University Press.

Yang, Y., Servedio, M. R., and Richards-Zawacki, C. L. (2019). Imprinting sets the stage for speciation. Nature 574, 99-102. doi: 10.1038/s41586-01 9-1599-z

Yorzinski, J. L. (2017). The cognitive basis of individual recognition. Curr. Opin. Behav. Sci. 16, 53-57. doi: 10.1016/j.cobeha.2017.03.009
Conflict of Interest: The authors declare that the research was conducted in the absence of any commercial or financial relationships that could be construed as a potential conflict of interest.

Copyright (c) 2020 Tumulty and Sheehan. This is an open-access article distributed under the terms of the Creative Commons Attribution License (CC BY). The use, distribution or reproduction in other forums is permitted, provided the original author(s) and the copyright owner(s) are credited and that the original publication in this journal is cited, in accordance with accepted academic practice. No use, distribution or reproduction is permitted which does not comply with these terms. 